ally into the central nervous system, discussed by Henson, the peripheral nervous system (Quay), the ear and audition (Henson), vision, olfaction and taste (Suthers) and finally to a brief account of the pineal by Quay. Other endocrine aspects will presumably follow later. The volume concludes with an enormous consideration (131 pages) of bats in relation to human health. While no one, least of all those who work with living bats, should doubt the importance of this subject, its place in this volume and the mystery about future plans suggest poor coherence in the whole work.

But for the rest we must (and will eagerly) wait and see. I have high hopes and whatever the outcome one thing seems certain: that the current increase in work on bats, which has occurred in spite of the lack of a book of this sort, will gain much impetus now that it has appeared. DAvid PyE

\section{Character and Attitude}

Character Structure and Impulsiveness. By David Kipnis. (Personality and Psychopathology: a Series of Monographs, Texts and Treatises.) Pp. xi+ 133. (Academic: New York and London, March 1971.) \$7.95; £3.75.

Attitude and Attitude Change. By Harry C. Triandis. (Wiley Foundations of Social Psychology Series.) Pp. xiii + 232. (Wiley: New York and London, April 1971.) $£ 3.50$ boards; $£ 1.65$ paper.

THE first of these books reports a series of interrelated studies, all based on the author's scale of impulsiveness, a scale which correlates both with Gough's socialization scale and the extraversion scale of the MPI. The scale concentrates on masculine outdoor hobbies, and all studies are therefore wisely limited to male subjects. Kipnis starts with the interesting and important idea that diagnostic instruments in psychology should be used like diagnostic tests in medicine, that is not only to describe the current state of a person but to show how to improve the score of those with a deficit on the test.

After a series of studies designed to establish the validity of his instrument he implements this action-orientation in the following way: high scorers on impulsiveness are underachievers in college examinations; they are also highly motivated by material incentives. Kipnis, accordingly established an ingenious field experiment with proper controls, in which impulsive underachievers were paid money for doing their mathematics course. The results show that the more intelligent impulsive students improved their grade in this condition, which had, however, no effect on the less intelligent. Although the role of intelligence as a moderator of other personality predispositions is interesting, it receives no theoretical treatment in this book, which is altogether deficient on theory. Furthermore, the effect disappeared when payment stopped. The intended "cure" may, for all one knows, only have strengthened the original "illness". Here, as elsewhere, nothing would have been more practical than a good theory to deal with such doubts.

The study of attitudes continues to dominate social psychology and social psychological publications. At least a dozen books on attitude and attitude change have been published during the past year. Triandis's contributionAttitude and Attitude Change-is in the textbook category. As one would expect from this author, it is workmanlike, clearly written and thoroughly up-todate. The text is refreshingly catholic in approach and goes beyond the currently most fashionable debate about consistency theories. Instructors and students will find the student assignments at the end of each chapter particularly useful; they are imaginative guides to self-instruction and encourage students to think about the underlying assumptions in attitude research. There is a bibliography of well over five hundred items. MARIE JAHODA

\section{Quantum Text}

Practical Quantum Mechanics I. By Siegfried Flügge. (Die Grundlehren der Mathematischen Wissenschaften in Einzeldarstellungen, Band 177.) Pp. xiv +341 . (Springer-Verlag: Berlin and New York, 1971.) 70 DM; $\$ 20.30$.

Practical Quantum Mechanics II. By Siegfried Flügge. (Die Grundlehren der Mathematischen Wissenschaften in Einzeldarstellungen, Band 178.) Pp. xii +287 .) (Springer-Verlag: Berlin and New York, 1971.) 60 DM; \$17.40.

ANYONE who has taught a course of quantum mechanics knows the difficulty of providing practical examples which are within the mathematical competence of the students and can be completed in a reasonable time. In these two volumes, which have been designed as a unified work, will be found 219 problems, together with their solutions, which will greatly extend the repertoire. They are all of moderate length and will be accessible to any student who can recognize the Bessel equation and consult references for properties of its solutions when these are required.

The first volume deals exclusively with one-body problems without spin. Many one dimensional and three dimensional potentials are introduced and the problems of calculating wavefunctions for bound states and for elastic scatter- ing are well represented. Applications are made to some specific situations in atomic and nuclear physics, with numerical values given to all the constants so as to emphasize the order of magnitude of the effects. The problems illustrating scattering resonances and Regge poles are especially welcome. In the second volume the problems cover a wider range and include illustrations of the introduction of spin, the interactions between two and three particles, quantum statistics and the Dirac relativistic equation with shorter sections on non-stationary problems and radiation theory. It will be very useful to have problems on tensor forces, the Tietz approximation in Thomas-Fermi theory and on helicity. Between the two volumes most of the features of elementary quantum mechanics which have to be compared with the corresponding features of classical mechanics are introduced and discussed.

Faced with so many useful problems and elegant solutions I am reluctant to turn to the limitations of this collection. It has to be said, however, that the problems are all of textbook type and deal with the solution of the differential equations for the wavefunctions and the subsequent calculation of properties. The classical techniques of mathematical analysis are emphasized. Matrix techniques, which are often better illustrations of real problems and are extensively used in modern self-consistent field treatments of atoms and molecules, scarcely appear and there is no group theory. The diagram techniques of modern perturbation theories appear briefly in only one solution. As an introduction to the practical problems of quantum theory and to the most used mathematical methods this is a very lopsided and unrepresentative compilation. As a preparation for courses on specialized aspects of quantum theory probably the examples on nuclear theory will be considered most adequate. The student interested in atomic and molecular physics, in field theory or in solid state physics will find few examples that he cannot find elsewhere.

The volumes have been well produced with many well designed graphs. The English style has some reminders of the German of the earlier edition, but the book has been rewritten. The purist will object to the abbreviation of "having no complete set of eigenvectors" to "having no eigenvectors" in some matrix problems.

For the teacher who selects carefully and can supplement from other sources, this will be a valuable and much used addition to the armoury. For the unaided student who learns best by working out examples and attempting open-ended problems the practical introduction to quantum mechanics still has to be written. G. G. Hall. 